PROCEEDINGS OF THE

AMERICAN MATHEMATICAL SOCIETY

Volume 131, Number 2, Pages 329-336

S 0002-9939(02)06824-7

Article electronically published on September 19, 2002

\title{
ON PRO-UNIPOTENT GROUPS SATISFYING THE GOLOD-SHAFAREVICH CONDITION
}

\author{
M. KASSABOV
}

(Communicated by Lance W. Small)

\begin{abstract}
We prove that a pro-unipotent group satisfying the Golod-Shafarevich condition contains a free non-abelian pro-unipotent group. Together with the result of A. Magid this implies that such a group is not linear.
\end{abstract}

\section{INTRODUCTION}

In [4] Golod and Shafarevich found a sufficient condition for a (pro- $p$ ) group represented by generators and relations to be infinite. Recently, Zelmanov [12] confirmed an earlier conjecture by Wilson [11] by showing that a pro- $p$ group satisfying the Golod-Shafarevich condition contains a free non-abelian pro- $p$ group.

In the early 80's Lubotzky and Magid (see [6, 5, 7]) developed a theory of prounipotent groups presented by generators and relators. They also extended the Golod-Shafarevich result to pro-unipotent groups.

In this article we prove that if $G$ is a pro-unipotent group satisfying the GolodShafarevich condition, then it contains a free pro-unipotent group (Theorem [5.3).

The proof in the pro- $p$ case cannot be translated directly to the pro-unipotent case because it relies on the fact that a free pro- $p$ group contains a countable dense subset, which is not true in the pro-unipotent case. To avoid this problem we need some facts about ideals in free Lie algebras.

This result can be combined with the result of Magid [7, who showed that a free pro-unipotent group is not linear. Together, these results imply that a prounipotent group satisfying the Golod-Shafarevich condition is not linear.

A large class of pro-unipotent groups satisfying the Golod-Shafarevich condition can be constructed from fundamental groups of 3-manifolds (see [2]).

The outline of this paper is as follows: $₫ 2$ gives the definition and basic properties of pro-unipotent and free pro-unipotent groups; $\$ 3$ presents the Golod-Shafarevich

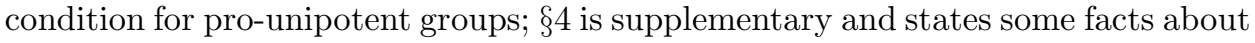
free Lie algebras which are used in the proofs that follow; $\$ 5$ gives the proof of the main result.

Received by the editors November 16, 2000.

2000 Mathematics Subject Classification. Primary 20E18; Secondary 17B65, 22 E65.

Key words and phrases. Pro-unipotent groups, Golod-Shafarevich condition. 


\section{Pro-Unipotent Groups}

Let $G$ be a pro-unipotent group, which is an inverse limit of a series $G_{i}$ of unipotent groups. A Lie algebra $\operatorname{Lie}(G)$ of $G$ is defined as a (projective) limit of Lie algebras corresponding to the groups $G_{i}$. There exists a map log: $G \rightarrow \operatorname{Lie}(G)$ and its inverse exp : $\operatorname{Lie}(G) \rightarrow G$, which satisfies the usual properties of the logarithm map in the case of unipotent Lie groups; for example, it is an isomorphism between pro-affine varieties. As in the case of nilpotent groups, it is convenient instead of working with the group to pass to (the completion of) the universal enveloping algebra of $\operatorname{Lie}(G)$. This algebra is denoted by $k\langle\langle G\rangle\rangle$, and is called a group algebra of $G$. In [ $[\underline{6}]$ it is proved that there exists a group embedding $\rho: G \hookrightarrow k\langle\langle G\rangle\rangle^{*}$, such that the image of $G$ generates $k\langle\langle G\rangle\rangle$ as a pro-nilpotent algebra. Moreover, if the group $G$ is generated by the elements $g_{i}$, then its group algebra $k\langle\langle G\rangle\rangle$ is generated by $s_{i}=\rho\left(g_{i}\right)-1$.

Let $G=U(X)$ be the free pro-unipotent group (the free object in the category of the pro-unipotent groups whose existence is proved in [5, 6]) on the finite set $X=\left\{g_{1}, \ldots, g_{n}\right\}$. Its group algebra, $k\langle\langle U(X)\rangle\rangle$, is isomorphic to $k\left\langle\left\langle s_{1}, \ldots, s_{n}\right\rangle\right\rangle$, the pro-nilpotent completion of the free associative algebra generated by $s_{i}$. Also, the group $U(X)$ can be considered as a subgroup of the multiplicative group of $k\left\langle\left\langle s_{1}, \ldots, s_{n}\right\rangle\right\rangle$.

For a more detailed introduction to pro-unipotent groups the reader is referred to [5, 6, 1].

\section{The Golod-Shafarevich COndition}

Let $\mathcal{G}$ be the category of pro-unipotent groups over a field $k$ of characteristic zero. Let $F_{\mathcal{G}}(n)$ denote the free (in $\mathcal{G}$ ) group on $n$ generators. Let $G$ be a finitely generated pro-unipotent group.

The commutator subgroups of $G$ form a filtration

$$
G=G_{1} \supset G_{2} \supset \cdots \supset G_{n} \supset \cdots
$$

defined by $G_{1}=G$ and $G_{i+1}=\overline{\left[G, G_{i}\right]}$. Here $\overline{[A, B]}$ is the closure of the subgroup generated by the commutators $[a, b]=a b a^{-1} b-1$ for $a \in A$ and $b \in B$. These subgroups form a basis of the pro-unipotent topology on $G$ and the group $G$ is complete in this topology.

This filtration satisfies the condition $\bigcap G_{i}=(1)$, since $G$ is a pro-unipotent group. Hence, every non-trivial element $g \in G$ falls into some gap $g \in G_{i} \backslash G_{i+1}$ for some unique $i$. We will call $i$ the degree of the element $g$ and denote it by $\operatorname{deg} g$. The Hilbert series of $G$ is defined using this filtration by $H_{G}(t)=\sum \operatorname{dim}\left(G_{i} / G_{i+1}\right) \cdot t^{i}$.

Consider a group $G=\left\langle x_{1}, \ldots, x_{n} \mid r=1, r \in R_{0}\right\rangle$ in the category $\mathcal{G}$ presented by generators $x_{i}$ and relators $R_{0} \subset F_{\mathcal{G}}$, i.e., $G=F_{\mathcal{G}} / N$, where $N$ is the minimal closed normal subgroup of $F_{\mathcal{G}}$ which contains $R_{0}$.

Without loss of generality, we may assume that $R_{0} \subset\left(F_{\mathcal{G}}\right)_{2}$, because relations in $\left(F_{\mathcal{G}}\right)_{1} \backslash\left(F_{\mathcal{G}}\right)_{2}$ are equivalent to an elimination of a generator. Define the Hilbert series of $R_{0}$ by $H_{R_{0}}(t)=\sum r_{i} t^{i}$, where $r_{i}$ is the number of elements in $R_{0}$ which have degree $i$.

Lubotzky and Magid [6] extended the Golod and Shafarevich result [4] (see also [9, 11]) from the case of discrete groups and associative algebras to unipotent 
groups. They proved that

$$
\frac{H_{G}(t)}{1-t}\left(1-n t+H_{R_{0}}(t)\right) \geq \frac{1}{1-t},
$$

where the inequality of formal series is termwise.

If there exists a real number $0<t_{0}<1$ such that $H_{R_{0}}\left(t_{0}\right)$ converges and

$$
1-n t_{0}+H_{R_{0}}\left(t_{0}\right)<0,
$$

we will say that the presentation for the group $G$ satisfies the Golod-Shafarevich condition. In this case $H_{G}\left(t_{0}\right)$ cannot converge; in particular, the group $G$ is infinite dimensional.

Remark 3.1. If the number of elements in $R_{0}$ is less than $n^{2} / 4$, the presentation satisfies the Golod-Shafarevich condition because $1-n t_{0}+H_{R_{0}}\left(t_{0}\right) \leq 1-n t_{0}+$ $n^{2} t_{0} / 4$ for all $0<t_{0}<1$, and the last expression is zero for some $t_{0}$.

For example, the pro-unipotent completion of the fundamental group of a 3-dimensional manifold satisfies the Golod-Shafarevich condition (except in few cases) because they are balanced, i.e., they have presentation with $n$ generators and $n$ relations (see [2]).

Remark 3.2. The Golod-Shafarevich condition was originally defined for associative algebras. A presentation of a pro-nilpotent algebra $A=\left\langle s_{i} \mid r=0, r \in R\right\rangle$ is said to satisfy the Golod-Shafarevich condition if there exists $0<t_{0}<1$ such that $H_{R}\left(t_{0}\right)$ converges and $1-n t_{0}+H_{R}\left(t_{0}\right)<0$.

Remark 3.3. Having the presentation of a pro-unipotent group $G$, we can obtain a presentation of its (pro-nilpotent) group algebra $k\langle\langle G\rangle\rangle$ in the following way: for each $r_{0} \in R_{0} \subset F_{\mathcal{G}}(X)$ we construct $r \in k\langle\langle T\rangle\rangle$ by $r=r_{0 \mid g_{i} \rightarrow 1+s_{i}}-1$, i.e., we substitute every generator $g_{i}$ with $1+s_{i}$ and expand the result as a series in the noncommutative variables $s_{i}$. Thus, we have that $\operatorname{deg} r=\operatorname{deg} r_{0}$. Let $R$ be the set of all $r$ for $r_{0} \in R_{0}$. The group algebra $k\langle\langle G\rangle\rangle$ has a presentation $k\left\langle\left\langle s_{i} \mid r=0, r \in R\right\rangle\right\rangle$ (see 6]). Therefore, the group algebra (with that presentation) satisfies the GolodShafarevich condition if and only if the group satisfies it. This result allows us to work mainly with the group algebra $k\langle\langle G\rangle\rangle$ for the remainder of the article.

The following lemma is a generalization of Golod's result (see 3]). In the case of a countable field, it was proven by Zelmanov in [12].

Lemma 3.4. For any $\epsilon>0$ and any $0<t_{0}<1$, there exists a subset $J$ of $K\left\langle s_{1}, \ldots, s_{m}\right\rangle$ and an increasing function $i \rightarrow h(i)$ such that:

(1) $H_{J}\left(t_{0}\right) \leq \epsilon$,

(2) every $i$ elements from the algebra $k\left\langle s_{1}, \ldots, s_{m} \mid v=0, v \in J\right\rangle$ of degree higher than $h(i)$ generate a nilpotent subalgebra.

Proof. A proof in the case of a countable field can be found in [12. That proof relies on the fact that the free algebra $k\langle X\rangle$ is a countable set. The proof that follows uses only that this algebra has a countable dimension, i.e., any element can be expressed as a finite linear combination of countably many basis elements.

Let $h: \mathbb{N} \rightarrow \mathbb{N}$ be an increasing function such that $r t_{0}^{h(r)}<1 / 4$ for any $r$. Let us fix $r$. Then, for any $l$, the expression $(N+r l)^{r l}\left(r t_{0}^{h(r)}\right)^{N}$ converges to 0 as $N$ tends to $\infty$. Therefore, for any integer $s \geq 1$, there exists a number $N_{r, l, s}$ such that

$$
\left(N_{r, l, s}+r l\right)^{r l}\left(r t_{0}^{h(r)}\right)^{N_{r, l, s}} \leq 2^{-(r+l+s)} \epsilon .
$$


Let $W_{n}$ be the set of all words on letters $s_{1}, \ldots, s_{m}$ of length greater than $n$. Also, denote by $\left(W_{h(r)}\right)^{(r, l)}$ the set of all $r l$-tuples of elements in $W_{n}$ of the form $\left\{w_{i, j}\right\}_{i=1, r}^{j=1, l}$. The set $\left(W_{h(r)}\right)^{(r, l)}$ is countable for any $r$ and $l$. Let $\gamma_{r, l}$ be a bijection from this set to $N$. Given an element $\alpha=\left\{w_{i, j}\right\}$, we can define the formal sum $b(\alpha)_{p}$ as $b(\alpha)_{p}=\sum_{j} t_{p, j} w_{p, j}$, where $\left\{t_{p, j}\right\}$ are formal commuting variables.

Let $\alpha=\gamma_{r, l}^{-1}(s) \in\left(W_{h(r)}\right)^{(r, l)}$ be an element such that $\gamma_{r, l}(\alpha)=s$. Let $u$ be a word of length $N_{r, l, s}$ composed of letters $y_{1}, \ldots, y_{r}$. Then, for any such word, denote by $J_{(\alpha, u)}$ the set of the coefficients of the monomial in $t_{i, j}$ in the expansion of

$$
u_{\mid y_{i} \rightarrow b(\alpha)_{i}}
$$

which is just a product of length $N_{r, l, s}$ on $b(\alpha)_{i}$-es. The set $J_{\alpha, u}$ contains less than $\left(N_{r, l, s}+r l\right)^{r l}$ elements (since this is greater than the number of monomials of degree $N_{r, l, s}$ of $r l$ commuting variables), and each element is of degree greater than $N_{r, l, s} h(r)$. Let

$$
J_{\alpha}=\bigcup_{u} J_{(\alpha, u)}, \quad J_{r, l}=\bigcup_{\alpha \in\left(W_{h(r)}\right)(l, r)} J_{\alpha}, \quad J_{r}=\bigcup_{l} J_{r, l}, \quad J=\bigcup_{r} J_{r} .
$$

Then,

$$
\begin{aligned}
& H_{J}\left(t_{0}\right) \leq \sum_{r, l, \alpha, u} H_{J_{(\alpha, u)}}\left(t_{0}\right)<\sum_{r, l, s} r^{N_{r, l, s}}\left(N_{r, l, s}+r l\right)^{r l} t_{0}^{N_{r, l, s} h(r)} \\
& \leq \sum_{r, l, s} 2^{-r-l-s} \epsilon=\left(\sum_{r} 2^{-r}\right)^{3} \epsilon=\epsilon .
\end{aligned}
$$

It remains to be shown that the set $J$ satisfies the conditions of the lemma. Let $y_{1}, \ldots, y_{r}$ be $r$ elements in $k\left\langle s_{1}, \ldots, s_{m}\right\rangle$, each of degree greater than $h(r)$. Then there exists $l$ such that each element can be expressed as a linear combination of at most $l$ monomials, i.e.,

$$
y_{i}=\sum_{j=1}^{l} t_{i, j} w_{i, j}
$$

where $w_{i, j}$ are monomials and $t_{i, j}$ are elements of the field $k$. Let $\alpha=\left\{w_{i, j}\right\}$ be the element in $\left(W_{h(r)}\right)^{l, r}$ corresponding to the monomials $w_{i, j}$. Then $y_{i}$ can be obtained from $b(\alpha)_{i}$ by substituting $t_{i, j}$-es with suitable elements from the field $k$. By construction, any product of length $N_{r, l, \gamma_{k, l}(\alpha)}$ on $b(\alpha)_{i}$-es is in the ideal generated by the set $J$, i.e., any product of this length on $y_{i}$-es lies in $\langle J\rangle$. Therefore, every $r$ elements of degree greater than $h(r)$ generate a nilpotent subalgebra of $k\left\langle s_{1}, \ldots, s_{m} \mid v=0, v \in J\right\rangle$.

\section{Some facts about free Lie algebras}

Let $\mathcal{L}[X]$ be the free Lie algebra generated by the set $X$. Define the ideals $I_{n}$ by $I_{0}=\mathcal{L}$ and $I_{n+1}=\left[\mathcal{L}, I_{n}\right]$. These ideals form a basis of the pro-nilpotent topology on $\mathcal{L}[X]$ and its completion in this topology is denoted by $\mathcal{L}[[X]]$. Each nonzero element $f \in \mathcal{L}[[X]]$ lies in $I_{n} \backslash I_{n+1}$ for some $n$, which is called the degree of $f$ and denoted by $\operatorname{deg} f$. The homogeneous component of $f$ of degree $n$ is called the leading term.

The algebra $\mathcal{L}[[X]]$ is a non-compact topological algebra and its topology is equivalent to the one defined by the norm $\|f\|=\exp (-\operatorname{deg} f)$. 
Let $d(n)$ be the dimension of the space of homogeneous elements of degree $n$ in the free Lie algebra $\mathcal{L}[x, y]$. There is an explicit formula for $d(n)$ which can be found in [8].

Lemma 4.1. There exists a nonzero element $l_{n}$ in the free Lie algebra

$$
\mathcal{L}\left[x_{1}, \ldots, x_{n}, y\right]
$$

which is poly-linear and skew symmetric in the $x_{i}$-es.

Proof. Let $l_{n}$ be the element

$$
l_{n}\left(x_{1}, \ldots, x_{n}, y\right)=\sum_{\sigma \in S_{n}}(-1)^{\sigma}\left[y, x_{\sigma(1)}, x_{\sigma(2)}, \ldots, x_{\sigma(n)}\right],
$$

where $\left[z_{1}, z_{2}, \ldots, z_{k}\right]$ is the left normed commutator of the elements $z_{1}, \ldots, z_{k}$ defined inductively by $\left[z_{1}, z_{2}, \ldots, z_{k}\right]=\left[\left[z_{1}, z_{2}, \ldots, z_{k-1}\right], z_{k}\right]$.

By construction, the element $l_{n}$ is poly-linear and skew symmetric in $x_{i}$. This element is not zero because in the lexicographic order such that $y>x_{1}>x_{2}>$ $\cdots>x_{n}$, its leading term (in the free associative algebra) is $y x_{1} x_{2} \ldots x_{n}$.

Remark 4.2. In the decompositon of $\mathcal{L}\left[x_{i}, y\right]$ under the natural action of the group $G L_{n+1}$, there is a unique submodule which corresponds to the partition $\left(2,1^{n-1}\right)$. This shows that the element $l_{n}$ is unique up to a scalar multiplication.

Lemma 4.3. For an arbitrary integer $n$, there exists an element $f_{n} \in \mathcal{L}[x, y]$ with the following property: Let $x, y \in L$ be elements of a Lie algebra $L$ such that there exists a homogeneous element $g \in \mathcal{L}[x, y]$ of degree $n$ for which the condition $g(x, y)=0$ in L holds. Then $f_{n}(x, y)=0$ in $L$.

Proof. If $n=1$ or $n=2$ we may take $f_{1}=f_{2}=[x, y]$, which is the only homogeneous Lie polynomial of degree 2 and, therefore, has the property in the lemma.

Let $n>2$. Let the elements $g_{1}, \ldots, g_{d(n)}$ form a basis of the space of homogeneous elements of degree $n$ in the free Lie algebra $\mathcal{L}[x, y]$.

Using degree arguments one can see that none of the elements $x, g_{i}$ can be expressed as a Lie polynomial of the others. This implies that elements $x, g_{i}$ freely generate a Lie subalgebra. Hence, there is no non-trivial Lie polynomial of $x, g_{i}$ which is zero in $\mathcal{L}[x, y]$ (see [8]). Therefore, the element

$$
f_{n}=l_{d(n)}\left(g_{1}, \ldots, g_{d(n)}, x\right)
$$

is nonzero. (Here, $l_{d(n)}$ is the element from Lemma 4.1])

If elements $x$ and $y$ in the Lie algebra $L$ satisfy the condition of this lemma, then the elements $g_{i}(x, y)$ are linearly dependent in $L$. Therefore, any skew symmetric expression of them vanishes, which implies that $f_{n}(x, y)=0$ in $L$.

Remark 4.4. The statement of the lemma is equivalent to the following: Let $h \in$ $\mathcal{L}[x, y]$ be a homogeneous element of degree $n$. Then $f_{n}$ lies in the ideal generated by $h$.

Lemma 4.5. Let $J$ be a homogeneous ideal of $\mathcal{L}[x, y]$. Then $J$ contains the ideal $\left\langle f_{n}\right\rangle$ for some $n$. 


\section{ON PRO-UNIPOTENT GROUPS SATISFYING THE GOLOD-ShaFAREVICH CONDITION}

Let $G=\left\langle g_{1}, \ldots, g_{m} \mid R_{0}=0\right\rangle$ be a pro-unipotent group satisfying the GolodShafarevich condition. Therefore, there exists a real number $t_{0}$ such that $H_{R_{0}}\left(t_{0}\right)<$ 0 . Let $p$ be an integer which satisfies $H_{R_{0}}\left(t_{0}\right)+2 t_{0}^{p}<0$ (such an integer exists since $\left.t_{0}<1\right)$.

Let $L$ be the Lie algebra of $G$ and let $A$ be the group algebra

$$
k\langle\langle G\rangle\rangle=k\left\langle\left\langle s_{1}, \ldots, s_{m} \mid r=0, r \in R\right\rangle\right\rangle .
$$

Thus, $L \hookrightarrow A^{-}$and $L$ is the Lie subalgebra in $A$ generated by the generators $s_{i}$ of $A$ (see [6]).

Assume that $G$ does not contain a free pro-unipotent group. Then $L$ does not contain a free (pro-nilpotent) Lie algebra $\mathcal{L}[[x, y]]$, i.e., for any $l_{1}, l_{2} \in L$, there exists an element $m_{\left(l_{1}, l_{2}\right)} \in \mathcal{L}[[x, y]]$ such that $m_{\left(l_{1}, l_{2}\right)}\left(l_{1}, l_{2}\right)=0$.

For any homogeneous Lie polynomial $h$, let $(L \times L)_{h}$ denote the set of all pairs $\left(l_{1}, l_{2}\right) \in L \times L$ such that there exists an $h^{\prime} \in \mathcal{L}[[x, y]]$ with $\operatorname{deg} h^{\prime}>\operatorname{deg} h$ and $h\left(l_{1}, l_{2}\right)=h^{\prime}\left(l_{1}, l_{2}\right)$ in $L$. This set is closed in the pro-nilpotent topology on $L$. Since every element in $\mathcal{L}[[x, y]]$ has a leading term, we have that

$$
(L \times L)=\bigcup_{h}(L \times L)_{h} .
$$

Let $\bar{I}_{i}$ be the ideal in $L$ which is the image of the ideal $I_{i} \subset \mathcal{L}\left[\left[s_{1}, \ldots, s_{m}\right]\right]$ for any integer $i$.

The first step in the proof is to show that the set $(L \times L)_{h}$ cannot contain an open subset of $\left(\bar{I}_{p} \times \bar{I}_{p}\right) \subset(L \times L)$. The proof is by contradiction.

Lemma 5.1. For any homogeneous element $h$ of degree $N$ in $\mathcal{L}[x, y]$, the set $(L \times L)_{h}$ does not contain an open subset of $\left(\bar{I}_{p} \times \bar{I}_{p}\right)$.

Proof. Assume that for some $h$ we have the inclusion

$$
\left(x_{0}+\bar{I}_{q}\right) \times\left(y_{0}+\bar{I}_{q}\right) \subset(L \times L)_{h},
$$

where $x_{0}, y_{0} \in \bar{I}_{p}$, and $q \geq p$ (since every open subset of $\left(\bar{I}_{p} \times \bar{I}_{p}\right)$ contains a subset of this type). Then the factor algebra $A^{\prime}=A /\left\langle x_{0}, y_{0}\right\rangle$ also satisfies the GolodShafarevich condition because the inequality $H_{R_{0} \cap\left\{x_{0}, y_{0}\right\}}(t) \leq H_{R_{0}}+2 t^{p}$ holds for all $t<1$, and the last expression is less than 0 for $t=t_{0}$. Let $L^{\prime}$ denote the Lie subalgebra of $A^{\prime}$ generated by the images of $s_{i}$ (the generators of $A$ ). This subalgebra is the image of $L$ under projection from $A$ to $A^{\prime}$.

The assumption on $(L \times L)_{h}$ implies that if $x, y$ are elements of $L^{\prime} \cap A^{\prime(q)}$, then there exists some $h^{\prime} \in \mathcal{L}$ such that $\operatorname{deg} h^{\prime}>N$ and such that $h(x, y)=h^{\prime}(x, y)$.

Let $f$ be the full linearization of some (non-trivial) poly-homogeneous component of $h$. It is known (see [1]) that $f\left(z_{1}, \ldots, z_{N}\right)$ can be expressed as a linear combination of values of $h$ in which $x$ and $y$ have been substituted by some linear combinations of the $z_{i}$-es. Therefore, for any $z_{i} \in L^{\prime} \cap A^{\prime(q)}$, there exists a $f^{\prime} \in \mathcal{L}\left[\left[z_{i}\right]\right]$ of degree greater than $N$ such that $f\left(z_{1}, \ldots, z_{n}\right)=f^{\prime}\left(z_{1}, \ldots, z_{n}\right)$.

Let $A^{\prime \prime}$ be the factor algebra of $A^{\prime}$ obtained by adding relations obtained from Lemma 3.4. Then the algebra $A^{\prime \prime}$ satisfies the Golod-Shafarevich condition together with the following conditions:

(1) The algebra $A^{\prime \prime}=\left\langle s_{1}, \ldots, s_{m}\right\rangle$ is a finitely generated associative algebra. 
(2) For all $i$, there exists an $h(i)$ such that every $i$ elements from $A^{\prime \prime h(i)}$ generate a nilpotent subalgebra.

(3) Let $L^{\prime \prime}$ be the Lie subalgebra of $A^{\prime \prime}$ generated by $s_{i}$. If we substitute $z_{i}$ with $l_{i} \in L^{\prime \prime} \cap A^{\prime \prime}(q)$ in $f$, we have $f\left(l_{1}, \ldots, l_{N}\right)=f^{\prime}\left(l_{1}, \ldots, l_{N}\right)$ for some $f^{\prime}$ in $\mathcal{L}\left[\left[z_{i}\right]\right]$ of degree $>N$.

In [12] it is proved that if an algebra satisfies the above conditions (1)-(3), then it is finite dimensional. Using this result we obtain a contradiction since the algebra $A^{\prime \prime}$ was constructed to satisfy the Golod-Shafarevich condition.

This Lemma shows that for each $h$ the set $(L \times L)_{h} \cap\left(\bar{I}_{p} \times \bar{I}_{p}\right)$ is a Baire set of first category because it is a closed nowhere dense set. Next, we want to apply Baire's theorem to the family of sets $\left\{(L \times L)_{h} \cap\left(\bar{I}_{p} \times \bar{I}_{p}\right)\right\}$. But this family is not countable, because the set of all homogeneous polynomials is not countable. Therefore, we need to choose a countable sub-family whose union is still the whole set $(L \times L)$.

Lemma 5.2. Let $h$ be a homogeneous polynomial of degree $N$. Then

$$
(L \times L)_{h} \subset(L \times L)_{f_{N}},
$$

where $f_{N}$ is the element from Lemma 4.3.

Proof. From Remark 4.4 we know that $f_{N}$ lies in the ideal of $\mathcal{L}[x, y]$ generated by $h$, i.e.,

$$
f_{N}=\sum_{w=w_{1} \ldots w_{k}} a_{w} h \operatorname{ad}\left(w_{1}\right) \operatorname{ad}\left(w_{2}\right) \ldots \operatorname{ad} w_{k},
$$

where the sum is over the words on letters $x, y$.

Let $(u, v) \in(L \times L)_{h}$. Then, there exists $h^{\prime}$ such that $h(u, v)=h^{\prime}(u, v)$ and $\operatorname{deg} h^{\prime}>N$. If we substitute this equality in the right side of the above expression for $f_{N}$, we obtain that $f_{N}(u, v)=f^{\prime}(u, v)$, where $\operatorname{deg} f^{\prime}>\operatorname{deg} f_{N}$. This shows that $(u, v) \in(L \times L)_{f_{N}}$.

From the previous lemma together with assumption that $G$ does not contain a free subgroup, we have that

$$
L \times L=\bigcup_{h}(L \times L)_{h}=\bigcup_{N}(L \times L)_{f_{N}} .
$$

But this is impossible by Baire's Category Theorem (see [10]), because $\left(\bar{I}_{p} \times \bar{I}_{p}\right)$ is a set of second category and by Lemma 5.1 the set $\left(\bar{I}_{p} \times \bar{I}_{p}\right) \cap(L \times L)_{f_{N}}$ is of first category for each $N$.

Thus, we have proven:

Theorem 5.3. Let $G$ be a pro-unipotent group which satisfies the Golod-Shafarevich condition. Then $G$ contains a free pro-unipotent group.

In fact we proved a slightly stronger result:

Corollary 5.4. Let $G$ be as above. Then a set of pairs $(x, y)$ such that $x, y$ does not generate a free pro-unipotent group inside $G$ form a Baire set of second category.

Using Magid's result ([7]) and Theorem [5.3 it is easy to obtain the following corollary. 
Corollary 5.5. Let $G$ be a pro-unipotent group which satisfies the Golod-Shafarevich condition. Then $G$ does not contain a linear (over any local field) subgroup of finite codimension.

The author thanks his advisor, E. Zelmanov, for formulating the problem.

\section{REFERENCES}

1. Vesselin Drensky, Free algebras and PI-algebras, Springer-Verlag Singapore, Singapore, 2000. MR 2000j: 16002

2. Michael Freedman, Richard Hain, and Peter Teichner, Betti number estimates for nilpotent groups, Fields Medalists' lectures, World Sci. Publishing, River Edge, NJ, 1997, pp. 413-434. MR 99d:20052

3. E. S. Golod, On nil-algebras and finitely approximable p-groups, Izv. Akad. Nauk SSSR Ser. Mat. 28 (1964), 273-276. MR 28:5082

4. E. S. Golod and I. R. Šafarevič, On the class field tower, Izv. Akad. Nauk SSSR Ser. Mat. 28 (1964), 261-272. MR 28:5056

5. Alexander Lubotzky and Andy R. Magid, Free prounipotent groups, J. Algebra 80 (1983), no. 2, 323-349. MR 85b:14062

6. Cohomology, Poincaré series, and group algebras of unipotent groups, Amer. J. Math. 107 (1985), no. 3, 531-553. MR 86h:20063

7. Andy R. Magid, Identities for prounipotent groups, Algebraic groups and their generalizations: classical methods (University Park, PA, 1991), Amer. Math. Soc., Providence, RI, 1994, pp. 281-290. MR 95j:20023

8. Christophe Reutenauer, Free Lie algebras, The Clarendon Press, Oxford University Press, New York, 1993. MR 94j:17002

9. Peter Roquette, On class field towers, Algebraic Number Theory (Proc. Instructional Conf., Brighton, 1965), Thompson, Washington, D.C., 1967, pp. 231-249. MR 36:1418

10. Walter Rudin, Functional analysis, McGraw-Hill Inc., New York, 1991. MR 92k:46001

11. John S. Wilson, Finite presentations of pro-p groups and discrete groups, Invent. Math. 105 (1991), no. 1, 177-183. MR 92k:20060

12. E. Zelmanov, On groups satisfying the Golod-Shafarevich condition, New horizons in pro- $p$ groups, Birkhäuser Boston, Boston, MA, 2000, pp. 223-232. MR 2002f:20037

Department of Mathematics, Yale University, 10 Hillhouse Ave., P.O. Box 208283, New Haven, Connecticut 06520-8283

E-mail address: martin.kassabov@yale.edu 